\title{
Molecular identification of the fish parasite Lernaea
}

\author{
B. PALLAVI, K. M. SHANKAR, P. B. ABHIMAN AND IQLAS AHMED \\ Aquatic Animal Health Laboratory, Department of Aquaculture, College of Fisheries Mangalore - 575002 \\ Karnataka, India \\ e-mail: kalkulishankar@gmail.com
}

\begin{abstract}
Isolates of the fish parasitic copepod Lernaea from four different freshwater fishes viz., catla (Catla catla), guppy (Poecilia reticulata), platy (Xiphophorus maculatus) and gold fish (Carassius auratus) were selected for the study. The isolates had considerably differing anchor shapes and were likely to be grouped as different species. Partial nucleotide sequences of the $18 \mathrm{~S}$ and 28S rDNA regions of the Lernaea isolates were determined. Phylogenetic trees generated by the neighbour joining method revealed that the Lernaea isolates examined in this study were of similar genotype and were identified to be Lernaea cyprinacea.
\end{abstract}

Keywords: Crustacean fish parasite, Lernaea, Molecular taxonomy, 18S rDNA, 28S rDNA

\section{Introduction}

Till date, 110 species of lernaeids have been systematically placed in 14 different genera (Ho, 1998). Currently 43 valid Lernaea species are reported in the World of Copepods database (Avenant, 2012). The taxonomical characteristics used for the identification of species of Lernaea often seem ill-defined. The shape of the anchors is selected as the most important characteristic for identification purposes. However, the growth of the anchors is largely influenced by the consistency of the tissue to which the parasite is attached ( $\mathrm{Hu}, 1948$; Harding, 1950; Fryer, 1961, 1968). Poddubnaya, (1973, 1978) further complicated the understanding of the species concept of lernaeids when she showed that offsprings with different phenotypes could be obtained from a single maternal specimen. The main contribution of her work is the demonstration that the morphology of the copepod may be substantially influenced by the host and that the influence of the host may be constant and predictable. Furthermore, it became evident from the work of Fratello and Sabatini (1972) that the chromosome of Lernaea collected from different fishes were identical and they concluded that all fishes carried the same species of Lernaea, i.e., L. cyprinacea. A total of nine species of Lernaea from India and South-east Asia have been reported so far. They are L. cyprinacea (Linnaeus, 1758), L. oryzophila (Monod, 1932), L. polymorpha (Yu, 1938), L. lophiara (Harding, 1950), L. chackoensis (Gnanamuthu, 1951), L. bengalensis (Gnanamuthu, 1956), L. arcuata (Soejanto, 1965), L. hesarghattensis (Srinivasachar and Sundarabai, 1974), L. bhadraensis (Seenappa et al., 1980) and L. osphronemi by Thomas et al. (1989). The considerable morphological plasticity of Lernaea species has hindered accuracy in identification (Kabata 1979; Lester and Haywood, 2006). Molecular genetic studies for definitive confirmation of species identity are a basic prerequisite for development of effective control strategies against the parasite.

The traditional taxonomical tools based on external morphology are often laborious, time consuming and require considerable skill. In contrast, molecular methods are relatively recent, reasonably accurate and could be complimented with other taxonomic methods. DNA sequence variations often contain useful information for taxonomic studies (Tautz et al., 2002, 2003; Blaxter and Floyd, 2003; Blaxter, 2004). The nuclear ribosomal DNA (rDNA) in eukaryotes is made up of tandem arrays of a basic unit that contains the transcription units (18S, $5.8 \mathrm{~S}, 28 \mathrm{~S}$ ) and an intervening intergenic spacer (IGS) region with copy numbers up to the order of 10,000 (Schlotterer, 1998). The ribosomal DNA cistron consists of two different regions; one region coding for ribosomal functioning (Raue et al., 1990) and others are non-coding. The different subunits and regions of the ribosomal DNA gene have different degrees of sequence variation and are suitable for comparison at the inter-genetic or interspecies level. The tick (Boophilus microplus) antigen Bm86, displays great variability among geographically isolated ticks leading to reduced effectiveness of the anti-tick vaccine (Kaewmongkol et al., 2015). Therefore, while designing a commercially viable vaccine against Lernaea it is prudent to ascertain if there is genetic variability among the Lernaea populations. Hence the present study was initiated to identify the Lernaea isolates using molecular tools. 


\section{Materials and methods}

\section{Collection of Lernaea isolates}

The Lernaea samples were collected from four different species of infected fishes viz., catla (Catla catla), guppy (Poecilia reticulata), gold fish (Carassius auratus) and platy (Xiphophorus maculatus) from the fish farm of the College of Fisheries, Mangalore, India. The parasite was pulled out with the help of forceps. Isolates from each host species was washed separately with $0.75 \% \mathrm{NaCl}$ and preserved in $95 \%$ ethanol until isolation of genomic DNA. The anchor shapes were studied under the microscope and confirmed that they were morphologically distinct from one another.

\section{$D N A$ extraction and polymerase chain reaction (PCR)}

Genomic DNA of the parasite was isolated using the Qiagen DNeasy Blood and Tissue kit as per the manufacturer's protocol. Purity and concentration of the DNA was checked by Nanodrop 2000C spectrophotometer (Thermo Scientific). The 18S rDNA fragments were amplified with primers $18 \mathrm{~S}$ F (5' - AAG GTG TGM CCT ATC AAC T - 3') and 18S R (5' - TTA CTT CCT CTA AAC GCT C - 3') designed by Song et al. (2008). The 28S rDNA fragments were amplified with primers $28 \mathrm{~S} \mathrm{~F}$ (5' - ACA ACT GTG ATG CCC TTA G - 3') and 28S R (5' - TGG TCC GTG TTT CAA GAC G - 3') designed by Song et al. (2008). PCR reactions were performed in $30 \mu \mathrm{l}$ mixtures containing $200 \mathrm{ng}$ genomic DNA, $0.2 \mu \mathrm{Mol}$ each of the two primers, $50 \mu \mathrm{M}$ of each of the dNTPs, $1 \times$ PCR buffer (with $1.5 \mathrm{mM} \mathrm{MgCl}$ ), $2.5 \mathrm{U}$ of Taq DNA polymerase (Genei, Bangalore, India) in BioRad C 1000 Touch Thermal Cycler under the following conditions: $94^{\circ} \mathrm{C}$ for $5 \mathrm{~min}$, followed by 30 cycles of $94^{\circ} \mathrm{C}$ for $30 \mathrm{~s}$, $54^{\circ} \mathrm{C}$ for $30 \mathrm{~s}$ and $72^{\circ} \mathrm{C}$ for $1 \mathrm{~min}$ with a final extension at $72^{\circ} \mathrm{C}$ for $5 \mathrm{~min}$. PCR reactions for $28 \mathrm{~S}$ regions were performed under similar conditions except that the annealing temperature was maintained at $60^{\circ} \mathrm{C}$ for $25 \mathrm{~s}$, extension at $72^{\circ} \mathrm{C}$ for $30 \mathrm{~s}$ with a final extension at $72^{\circ} \mathrm{C}$ for $3 \mathrm{~min}$. The amplified fragments were visualised on $1 \%$ agarose gel.

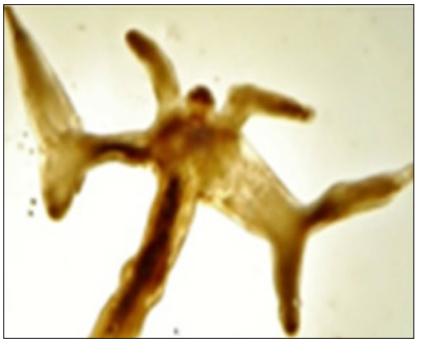

(a)

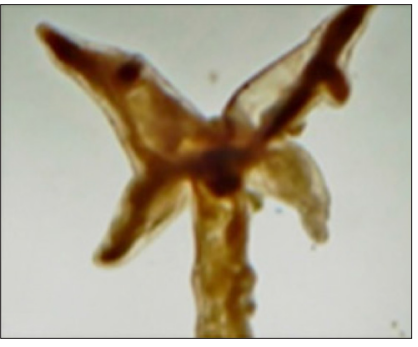

(b)

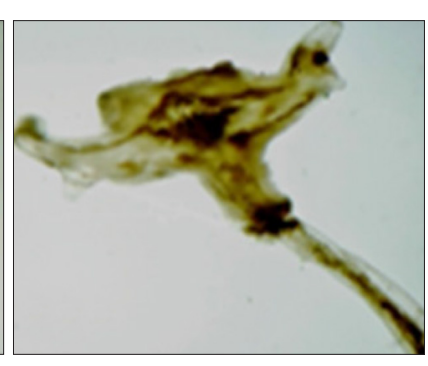

(c)

\section{DNA sequencing and assembly}

Specific amplified products were eluted from the gel using the Thermo Scientific Gene JET gel extraction and DNA cleanup microkit. Purified products were sent to Chromous Biotech, Bangalore, India for automated sequencing. The sequence was manually checked and edited for accuracy using Bioedit Software. Contigs were assembled using the CAP3 interface in the bioedit software.

\section{Phylogenetic analysis}

Lernaea cyprinacea sequences deposited by Song et al. (2008) and the Argulus sequences to be used as outgroup were downloaded from Genbank (http://www. ncbi.nih.gov) in the FASTA format. The sequences were then aligned with homologous sequences of the isolates using 'Clustal W' alignment software (Larkin et al., 2007) in MEGA version 5 (Tamura et al., 2011) (http://www. megasoftware.net).

The evolutionary history for both $18 \mathrm{~S}$ and $28 \mathrm{~S}$ sequences from Lernaea isolates was inferred using the Neighbor-Joining method (Saitou and Nei, 1987). The percentage of replicate trees in which the associated taxa were clustered together was calculated with the bootstrap test (1000 replicates) (Felsenstein, 1985). The evolutionary distances were computed using the Kimura 2- parameter method (Kimura, 1980) and are in the units of the number of base substitutions per site. The analysis involved 7 nucleotide sequences. All positions with less than $95 \%$ site coverage were eliminated. Fewer than 5\% alignment gaps, missing data and ambiguous bases were allowed at any position. There were a total of 1015 positions in the final dataset for the $18 \mathrm{~S}$ region and 662 positions for $28 \mathrm{~S}$ region.

\section{Results and discussion}

The anchor shapes of the Lernaea isolates from the four fishes considerably differed from each other (Fig. 1a, b, c and d). Polymerase chain reaction of the 18S rDNA region of Lernaea isolates yielded a product of $1370 \mathrm{bp}$ and that of $28 \mathrm{~S}$ region yielded a product

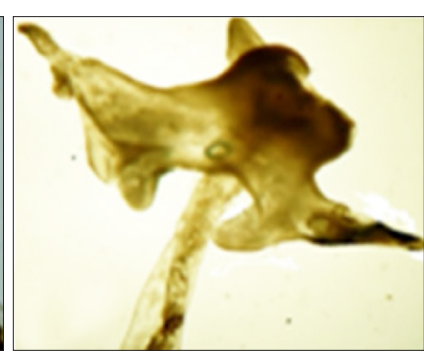

(d)

Fig. 1. Anchor of Lernaea isolated from a) Catla; b) Guppy; c) Platy; d) Gold Fish (x100) 
of $720 \mathrm{bp}$ (Fig. 2 and 3). The BLAST results showed that the sequences for the $18 \mathrm{~S}$ and $28 \mathrm{~S}$ rDNA from the Lernaea isolates were identical to the Lernaea cyprinacea sequences deposited in Genbank (Song et al., 2008). The sequencing results are presented in Fig. 4 and 5. Phylogenetic analyses were conducted in MEGA5 (Tamura et al., 2011). The distance trees generated by neighbor joining method reflect these findings (Fig. 6

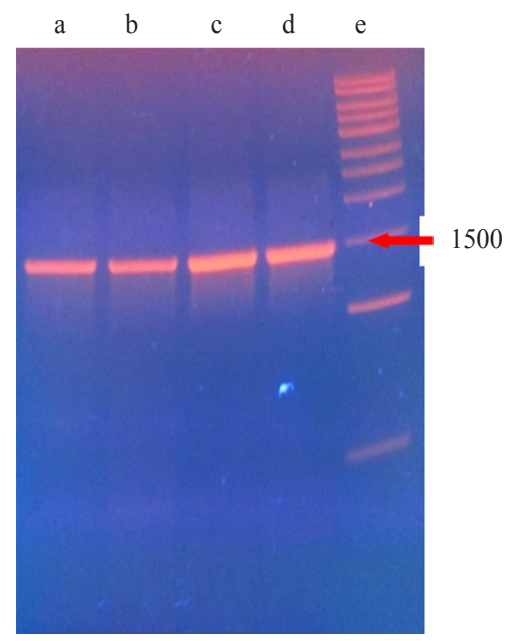

Fig. 2. Photograph of agarose gel containing PCR products of the 18S rDNA region of Lernaea isolates Lane a: Catla, b: Guppy, c: Platy, d: Gold fish, e: Marker and 7). This is reliable as the outgroup species Argulus was clearly segregated into a different clade.

The traditional taxonomic method considers the anchor shape of Lernaea as the most distinguishing characteristic to delineate the species. Using anchor shape as the basis for classification, the Lernaea isolates in the present study were likely to be grouped into four different species. The anchor shape is not a good characteristic for

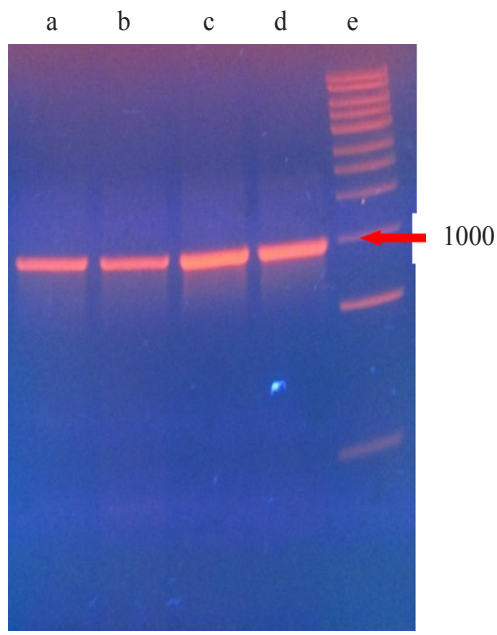

Fig. 3. Photograph of agarose gel containing PCR products of the 28S rDNA region of Lernaea isolates Lane a: Catla, b: Guppy, c: Platy, d: Gold fish

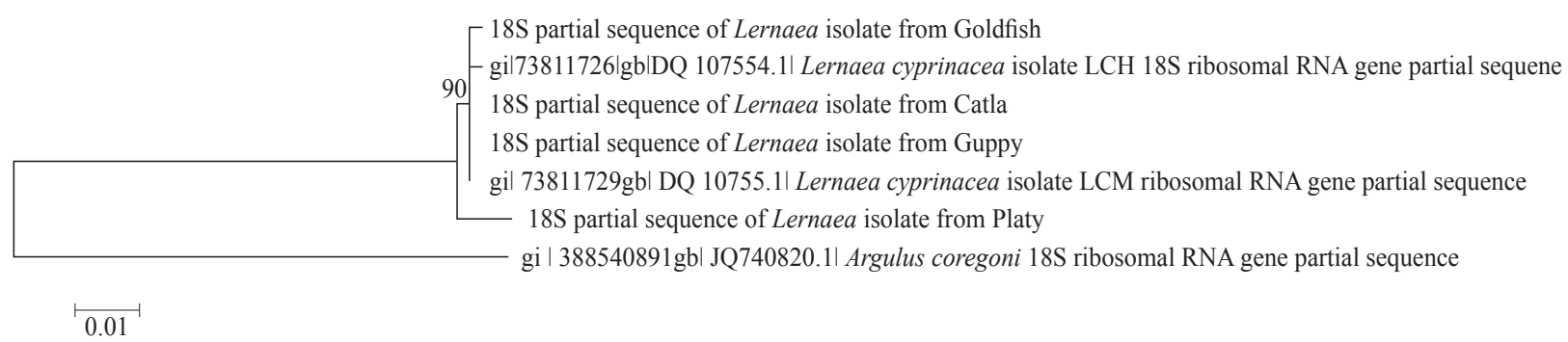

Fig. 6. Neighbor joining tree inferred from the analysis of partial 18S rDNA sequences of four Lernaea isolates. Numbers along branches indicate the percentages of support values. The optimal tree with the sum of branch length $=0.15845141$. The tree is drawn to scale, with branch lengths in the same units as those of the evolutionary distances used to infer the phylogenetic tree

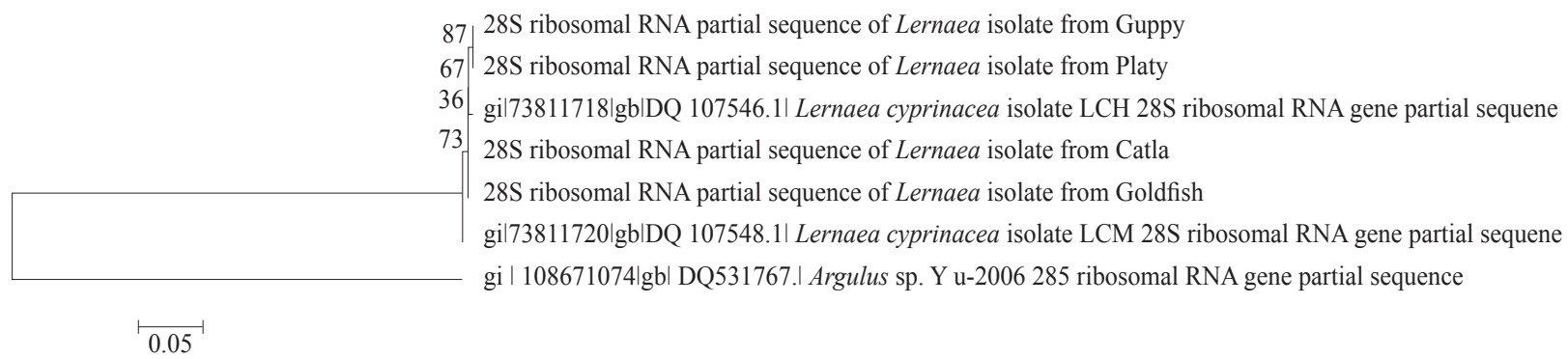

Fig. 7. Neighbor joining tree inferred from the analysis of partial $28 \mathrm{~S}$ rDNA sequences of four Lernaea isolates. Numbers along branches indicate the percentages of support values. The optimal tree with the sum of branch length $=0.63954580$. The tree is drawn to scale, with branch lengths in the same units as those of the evolutionary distances used to infer the phylogenetic tree 
$18 \mathrm{~S}$ rRNA Lernaea (catla) 18S rRNA Lernaea (guppy) 18S rRNA Lernaea (platy) 18S rRNA Lernaea (goldfish)

18S rRNA Lernaea (catla) 18S rRNA Lernaea (guppy) 18S rRNA Lernaea (platy) 18S rRNA Lernaea (goldfish) 18S rRNA Lernaea (catla) 18S rRNA Lernaea (guppy) 18S rRNA Lernaea (platy) 18S rRNA Lernaea (goldfish)

18S rRNA Lernaea (catla) 18S rRNA Lernaea (guppy) 18S rRNA Lernaea (platy) 18S rRNA Lernaea (goldfish)

18S rRNA Lernaea (catla) 18S rRNA Lernaea (guppy) 18S rRNA Lernaea (platy) 18S rRNA Lernaea (goldfish) 18S rRNA Lernaea (catla) 18S rRNA Lernaea (guppy) 18 S rRNA Lernaea (platy) 18S rRNA Lernaea (goldfish)

18S rRNA Lernaea (catla) 18S rRNA Lernaea (guppy) 18 S rRNA Lernaea (platy) 18S rRNA Lernaea (goldfish)

18S rRNA Lernaea (catla) 18S rRNA Lernaea (guppy) 18S rRNA Lernaea (platy)

18S rRNA Lernaea (goldfish)

18S rRNA Lernaea (catla) 18S rRNA Lernaea (guppy) 18S rRNA Lernaea (platy) 18S rRNA Lernaea (goldfish)

18S rRNA Lernaea (catla) 18S rRNA Lernaea (guppy) 18S rRNA Lernaea (platy) $18 \mathrm{~S}$ rRNA Lernaea (goldfish)

18S rRNA Lernaea (catla) 18S rRNA Lernaea (guppy) 18S rRNA Lernaea (platy) 18S rRNA Lernaea (goldfish)

18S rRNA Lernaea (catla) 18S rRNA Lernaea (guppy) 18S rRNA Lernaea (platy)

18S rRNA Lernaea (goldfish)

18S rRNA Lernaea (catla) 18S rRNA Lernaea (guppy) 18S rRNA Lernaea (platy)

18S rRNA Lernaea (goldfish)

18S rRNA Lernaea (catla) 18S rRNA Lernaea (guppy) 18S rRNA Lernaea (platy)

18S rRNA Lernaea (goldfish)

18S rRNA Lernaea (catla) 18S rRNA Lernaea (guppy) 18S rRNA Lernaea (platy) 18S rRNA Lernaea (goldfish)

18S rRNA Lernaea (catla) 18S rRNA Lernaea (guppy) 18S rRNA Lernaea (platy) 18S rRNA Lernaea (goldfish)

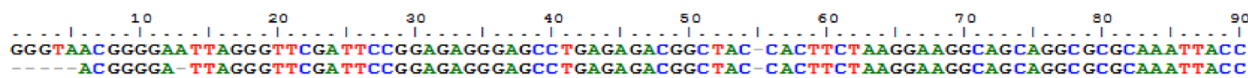

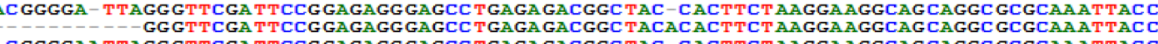

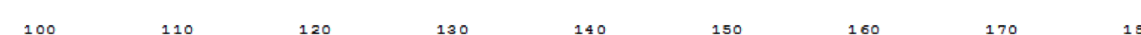
CACTGGCGAAGGCCGAGTAGTGACGAAAATAACGATACGGACTCATCGAGGCCCGGTAATCGGAATGAGTACACTTTAAATCCTT

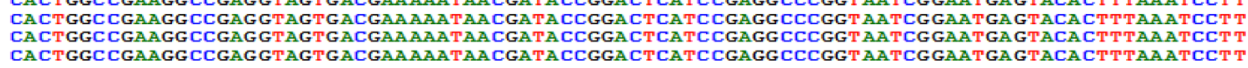

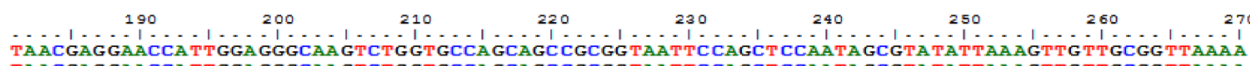

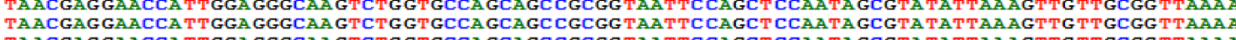

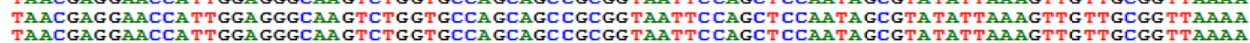

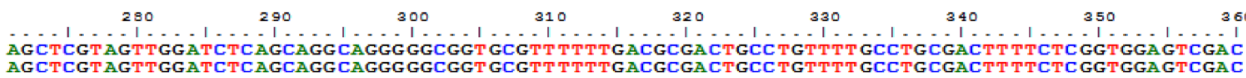

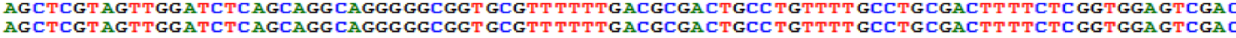

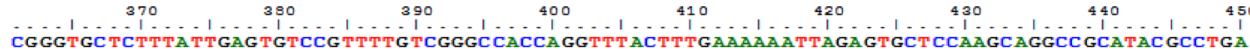
CGGGTGCTCTTPATTGAGTGTCCGTTTTGTCGGGCCACCAGGTTPACTTTGAAAAATTAGAGTGCT CCAAGCAGGCGCATACGCCTGA
CGGGTGCTCTTTATTGAGTGTCCGTTTGTCGGGCCACCAGGTTTACTTGAAAAAATTAGAGTGCTCCAAGCAGGCCGCATACGCCTGA CGGGT GC TCTTTA TTGAG TGTCCG TTTTG TCGGGCCACCAGGTTTACTTTGAAAAAAATTAGAGTGCTCCAAGCAGGCC GCATACGCCTGA

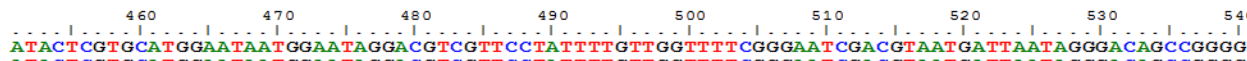
ATACTCGTGCATGGAATAATGGAATAGGACGTCGTTCCTATTTTGTTGGTTTT CGGGAAT CGACGTAATGATTAATAGGGACAGCCGGGC
ATACTCGTGCATGGAATAATGGAATAGGACGTCGTTCCTATTTTGTTGGTTTTCGGGAATCGACGTAATGATTAATAGGGACAGCCGGGG ATACTCGTGCATGGAATAATGGAATAGGACGTCGTTCCTATTTTGTTGGTTTTCGGGAATCGACGTAATGATTAATAGGGACAGCCGGGG

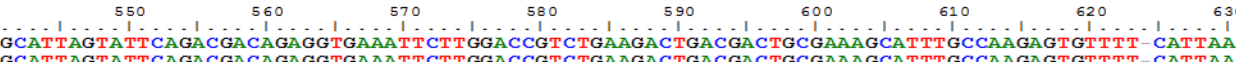
CATTAGTATTCAGACGACAGAGGTGAAATTCTTGGACCGTCTGAAGACTGACGACTGCGAAAGCATTTGCCAAAAGTGTTTTTCTTTAA GCATTAGTATTCAGACGACAGAGGTGAAATTCTTGGACCGTCTGAAGACTGACGACTGCGAAAGCATTTGCCAAGAGTGTTTT-CATTAA

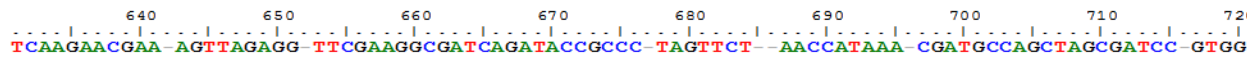
TCAAGAACGAA-AGTTAGAGG-TTCGAAGGCGATCAGATACCGCCC-TAGTTCT-AACCATAAA-CGATGCCAGCTAGCGATCC-GTGE TCAAGAACCAATAGT TAGAGGGTTCGAAGGCAATCAGATACCGCCCCTAGTTCCTAAACCATAAAACGATGCCAGCTAGCGATCCGTGGG
TCAAGAACGAA-AGTTAGAGG-TTCGAAGGCGATCAGATACCGCCC-TAGTCT--AACCATAAA-CGATGCCAGCTAGCGATCCGTGG

$\begin{array}{rrrrrrrr}730 & 740 & 750 & 760 & 770 & 780 & 790 & 800\end{array}$

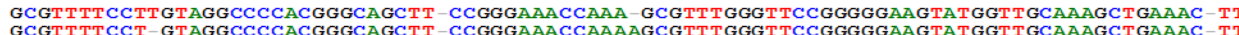
GCGTTTCCTTGTAGGCCCCACGGGCAGCTTTCCGGGAAACCAAA-GCGTTTGGGTTCCGGGGGAAGTATGGTTGCAAAGCTGAAACCTT

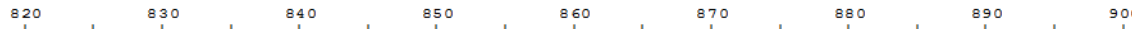

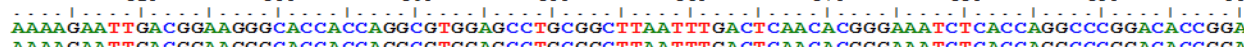
AAAAAATT GACGGAAGGCACCACCAGGCGTGGAGCCTGCGGCTTAATTTGACTCAACACGGGAATCTCACCAGGCCGGACACCGGA AAAAGAATTGACGGAAGGGCACCACCAGGCGTGGAGCCTGCGGCTTAATTTGACTCAACACGGGAAATCTCACCAGGCCCGGACACCGGA

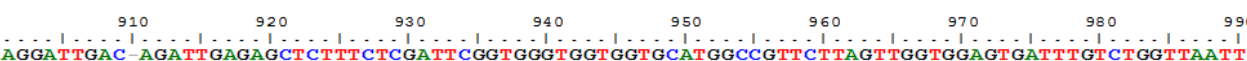
AGGATTGAC-AGATT GAGAGCTCTTTCTCGATTCGGTGGGTGGTGGTGCATGGCCGTTCTTAGTTGGTGGAGT GATTTGTCTGGTTAAT AGGATTGAC-AGATTGAGAGCTCTTTCTCGATTCGGTGGGTGGTGGTGCATGGCCTTCTTAGTTGGTGGAGTGATTTGTCTGGTTAATT
AGGATTGACGAGATT GAGAGCTCTTTCTCGATTCGGTGGGTGGTGTGCATGGCCGTTCTTAGTTGGTGGAGTGATT ATCTGGTTAATT

$\begin{array}{lllllllll}1000 & 1010 & 1020 & 1030 & 1040 & 1050 & 1060 & 1070 & 1080\end{array}$ CCGATAACGACGAGACTCCGTCTGCTAAATAGTGCCAGGTTGGTTTTTTTCCTACTCCTGGCGATGTCTCTTAGAGGAATG CCGATAACGAACGAGACTCCGTCCTGCTAAATAGTCGCCAGGTTGGTGTTTTTTTTCTACTGCCTGGCGAGTGTCTTCTTAGAGGGACTG CCGATAACGAACGAGACTCCGTCCTGCTAAATAGTCGCCAGGTTGGTGTTTTTTTTCTACTGCCTGGCGAGTGTCTTCTTAGAGGGACTG
CCGATAACGAACGAGACTCCGTCCTGCTAAATAGTCGCCAGGTTGGTTTTTTTTCTACTGCCTGGCGAGTGTCTTCTTAGAGGACTG

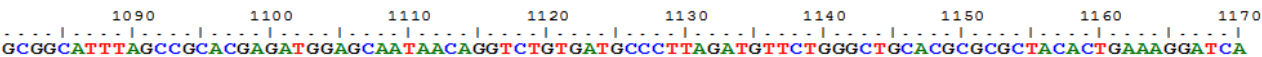
GCGGCATT TAGCCGCACGAGATGGAGCAATAACAGGTCTGTGATGCCCT TAGATGTTCTGGGCTGCACGCGCGCTACACTGAAAGGATCA GCGGCATTTAGCCGCACGAGATGGAGCAATAACAGGTCTGTGATGCCCTTAGATGTTCTGGGCTGCACGCGCGCTACACTGAAAGGATCA
GCGGCATTTAGCCGCACGAGATGGAGCAATAACAGGTCTGTGATGCCCTTAGATGTTCTGGGTGCACGCGCGCTACACT GAAAGGATCA

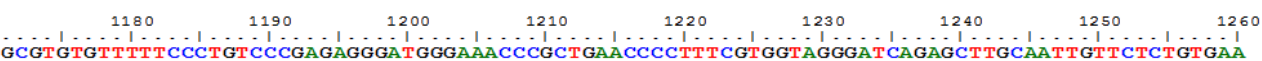
GCGTGTGTTTTTCCCT GTCCCGAGAGGGATGGGAAACCCGCTGAACCCC TTTCGT GGTAGGGATCAGAGCTTGCAATTGTTCTCTGTGA GCGTGTGTTTTTCCCTGTCCCGAGAGGATGGGAAACCCGCTGAACCCCTTTCGTGGTAGGGATCAGAGCTTGCAATTGTTCTCTGTGAA
GCGTGTGTTTTCCCTGTCCCGAGAGGGATGGGAAACCCGCTGAACCCCTTTCGTGGTAGGGATCAGAGCTTGCAATTGTTCTCTGTGAA

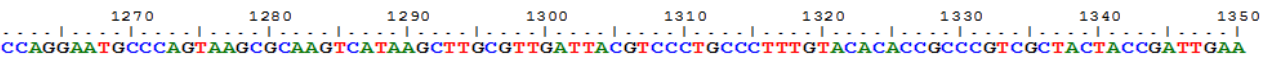
CAGGAATGCCCAGTAAGCGCAAGTCATAAGCTTGCGTTGATTACGTCCCTGCCCTTGACACACCGCCCGTCGCTACTACCGATTGA CCAGGAATGCCCAGIAAGCGCAATCATAAGCTIGCOHGATIACGH CGTTTTAGTAGGTATTTGGACGGGCCTGCGAAGTT CGTTT TAGTGAGGTATTTGGACTGGGCCTGCGGAA-

CGTTTTAGTGAGGTATTTGGACTGGGCCTGCGGAAC

Fig. 4. Clustal W alignment of nucleotide sequences of the $18 \mathrm{~S}$ rDNA regions of the Lernaea isolates from catla, guppy, platy and gold fish 
28S rRNA Lernaea (catla) $28 \mathrm{~S}$ rRNA Lernaea (guppy) $28 \mathrm{~S}$ rRNA Lernaea (platy)

28S rRNA Lernaea (goldfish)

28S rRNA Lernaea (catla) $28 \mathrm{~S}$ rRNA Lernaea (guppy) $28 \mathrm{~S}$ rRNA Lernaea (platy) 28S rRNA Lernaea (goldfish)

28S rRNA Lernaea (catla) $28 \mathrm{~S}$ rRNA Lernaea (guppy) 28S rRNA Lernaea (platy) $28 \mathrm{~S}$ rRNA Lernaea (goldfish)

28S rRNA Lernaea (catla) 28S rRNA Lernaea (guppy) 28S rRNA Lernaea (platy) 28S rRNA Lernaea (goldfish)

28S rRNA Lernaea (catla) 28S rRNA Lernaea (guppy) 28S rRNA Lernaea (platy)

28S rRNA Lernaea (goldfish)

28S rRNA Lernaea (catla) 28S rRNA Lernaea (guppy) $28 \mathrm{~S}$ rRNA Lernaea (platy) 28S rRNA Lernaea (goldfish)

28S rRNA Lernaea (catla) $28 \mathrm{~S}$ rRNA Lernaea (guppy) $28 \mathrm{~S}$ rRNA Lernaea (platy) $28 \mathrm{~S}$ rRNA Lernaea (goldfish)

28S rRNA Lernaea (catla) 28S rRNA Lernaea (guppy) 28S rRNA Lernaea (platy) $28 \mathrm{~S}$ rRNA Lernaea (goldfish)

28S rRNA Lernaea (catla) 28S rRNA Lernaea (guppy) 28S rRNA Lernaea (platy) 28S rRNA Lernaea (goldfish)

28S rRNA Lernaea (catla) $28 \mathrm{~S}$ rRNA Lernaea (guppy) $28 \mathrm{~S}$ rRNA Lernaea (platy) 28S rRNA Lernaea (goldfish)

28S rRNA Lernaea (catla) $28 \mathrm{~S}$ rRNA Lernaea (guppy) $28 \mathrm{~S}$ rRNA Lernaea (platy) 28S rRNA Lernaea (goldfish)

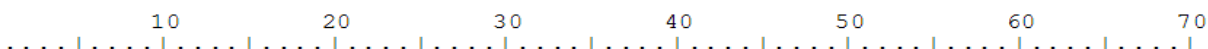
CCTTAGTAATGGCGAATGAAACGGGCAGAGCCCAGCACTGAACCGCCAGCTTTTGAAAGGGTTGCGCGG CCCTTAGTAATGGCGAATGAAACGGGCAGAGCCCAGCACTGAACCGCCAGCTTTTGAAAGGGTTGCGCGG СССTTAGTAATGGCGAATGAAACGGGCAGAGCCCAGCACTGAACCGCCAGCTTTTGAAAGGGTTGCGCGG CCCTTAGTAATGGCGAATGAAACGGGCAGAGCCCAGCACTGAACCGCCAGCTTTTGAAAGGGTTGCGCGG

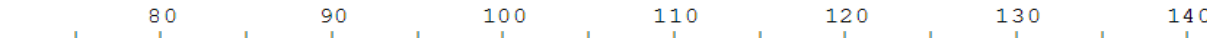
ÄTGTAGTGTTTGGGAGAGCCTTCTCATGATGCGCGGTCÄÄATTGTCTÄATCCACCTTGACTGGGG AATGTAGTGTTTGGGAGAGCCTTCTCATGATGCGCGGTGCAAAATCTGTCTAAGTCCACCTTGACTGGGG AATGTAGTGTTTGGGAGAGCCTTCTCATGATGCGCGGTGCAAAATCTGTCTAAGTCCACCTTGACTGGGG AATGTAGTGTTTGGAGAGCCTTCTCATGATGCGCGGTGCAAAATCTGTCTAAGTCCACCTTGACTGGGG

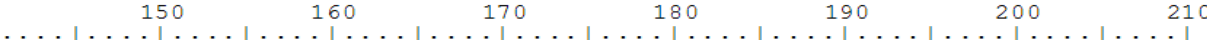
CCACTACCCATAGAGGTGATAGGCCCGTAAGACAGTCTGCGTGTTGTGCTGGCTTTTTCCCTAGAGTCG CCACTACCCATAGAGGGTGATAGGCCCGTAAGTGAGTCTGCGTGTTGTGCTGGCTTTTTCCCTAGAGTCG CCACTACCCATAGAGGGTGATAGGCCCGTAAGTGAGTCTGCGTGTTGTGCTGGCTTTTTCCCTAGAGTCG CCACTACCCATAGAGGGTATAGGCCCGTAAGACAGTCTGCGTGTTGTGCTGGCTTTTTCCCTAGAGTCG

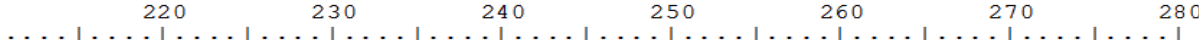

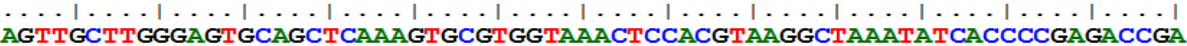
AGTTGCTTGGGAGTGCAGCTCAAAGTGCGTGGTAAACTCCACGTAAGGCTAAATATCACCCCGAGACCGA AGTTGCTTGGGAGTGCAGCTCAAAGTGCGTGGTAAACTCCACGTAAGGCTAAATATCACCCCGAGACCGA AGTTGCTTGGGAGTGCAGCTCAAAGTGCGTGGTAAACTCCACGTAAGGCTAAATATCACCCCGAGACCGA

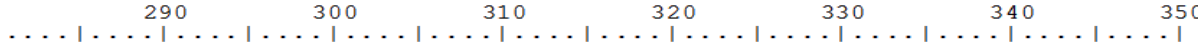
TAGCGAACAAGTACCGTGAGGGAAAGTTGAAAAGAACTTTGAAGAGAGAGTTCAATAGTACGTGAAACTG TAGCGAACAAGTACCGTGAGGGAAAGTTGAAAAGAACTTTGAAGAGAGAGTTCAATAGTACGTGAAACTG TAGCGAACAAGTACCGTGAGGGAAAGTTGAAAAGAACTTTGAAGAGAGAGTTCAATAGTACGTGAAACTG TAGCGAACAAGTACCGTGAGGGAAAGTTGAAAAGAACTTTGAAGAGAGAGTTCAATAGTACGTGAAACTG

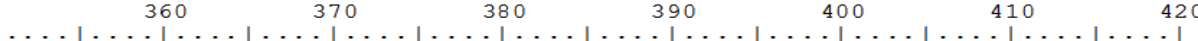
TGTAGCGGTAAACAGAGGGGCTCTCGAÄTCCAGGCTGGAGATTCAGGTTGCCAGATGGCTAGTTTGGCT TGTAGCGGTAAACAGAGGGGCTCTCGAAGTCCAGGCTGGAGATTCAGGTTGCCAGATGGCTAGTTTGGCT TGTAGCGGTAAACAGAGGGGCTCTCGAAGTCCAGGCTGGAGATTCAGGTTGCCAGATGGCTAGTTTGGCT TGTAGCGGTAAACAGAGGGGCTCTCGAAGTCCAGGCTGGAGATTCAGGTTGCCAGATGGCTAGTTTGGCT

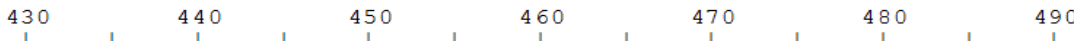

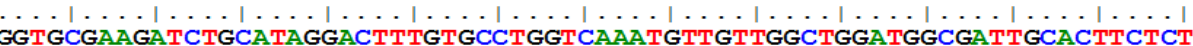
GGTGCGAAGATCTGCATAGGACTTTGTGCCTGGTCAAATGTTGTTGGCTGGATGGCGATTGCACTTCTCT GGTGCGAAGATCTGCATAGGACTTTGTGCCTGGTCAAATGTTGTTGGCTGGATGGCGATTGCACTTCTCT GGTGCGAAGATCTGCATAGGACTTTGTGCCTGGTCAAATGTTGTTGGCTGGATGGCGATTGCACTTCTCT

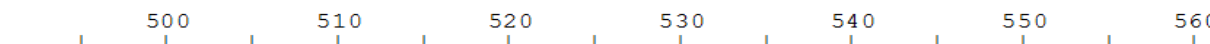
GGCCTAGCAATGGGCGCGACGAGCCACTGAGAGCGAATCAAGTGCGTGGGTGAAGTTTGCTTCAACAGTC GGCCTAGCAATGGGCGCGACGAGCCACTGAGAGCGAATCAAGTGCGTGGGTGAAGTTTGCTTCAACAGTC GGCCTAGCAATGGGCGCGACGAGCCACTGAGAGCGAATCAAGTGCGTGGGTGAAGTTTGCTTCAACAGTC GGCCTAGCAATGGGCGCGACGAGCCACTGAGAGCGAATCAAGTGCGTGGGTGAAGTTTGCTTCAACAGTC

\begin{tabular}{c|c|c|c|c|c|c|c|}
570 & 580 & 600 & 610 & 620 & 630
\end{tabular} $\ldots \ldots$ TTATGGTGGTGTTGTGAGCCCCGCGTTTTCTGGCTTCGATTTCGGTGGTCTTATGTATGGAGATAGGAC TTATGGCTGGTGTTGTGAGCCCCGCGTTTTCTGGCTTCGATTTCGGTGGTCTTATGTATGGAGATAGGAC TTATGGCTGGTGTTGTGAGCCCCGCGTTTTCTGGCTTCGATTTCGGTGGTCTTATGTATGGAGATAGGAC TTATGGCTGGTGTTGTGAGCCCCGCGTTTTCTGGCTTCGATTTCGGTGGTCTTATGTATGGAGATAGGAC

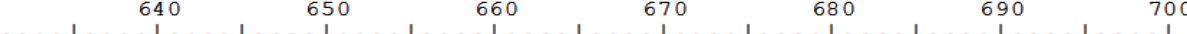
ÄACTCGTTTATAGCGAGTGCCGCTTTTGTGGCACTGTCTTTGTCCGACATCTGTCGCGAGTAGGTCGGT AGACTCGTTTATAGCGAGTGCCGCTTTTGTGGCACTGTCTTTGTCCGACATCTGTCGCGAGTAGGTCGGT AGACTCGTTTATAGCGAGTGCCGCTTTTGTGGCACTGTCTTTGTCCGACATCTGTCGCGAGTAGGTCGGT AGACTCGTTTATAGCGAGTGCCGCTTTTGTGGCACTGTCTTTGTCCGACATCTGTCGCGAGTAGGTCGGT

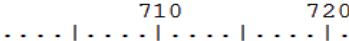

GGCTCTCTGACCCGTCTTGA

GGCСTCTCTGACCC

GGCCTCTCTGACCC-------

GGCCTCTCTGACCCGTCTT--

Fig. 5. Clustal W alignment of nucleotide sequences of the $28 \mathrm{~S}$ rDNA regions of the Lernaea isolates from catla, guppy, platy and gold fish 
identification of species as it is usually influenced by the type of fish tissue within which it developed. Very little sequence variability in the $18 \mathrm{~S}$ and $28 \mathrm{~S}$ rDNA regions supports that Lernaea parasitising the fishes in this study are monophyletic and might be the cosmopolitan species Lernaea cyprinacea itself. However, further analyses of genes of these isolates will be required before final conclusion. Other genes and a much diverse range of host fish infected with Lernaea need to be included. Further studies would help to understand whether the isolates share similar biological and epidemiological properties. Accidental introduction of $L$. cyprinacea might have occurred in the water bodies of Mangalore most probably as a result of the import of tropical ornamental fish from different parts of Asia. The host fishes of Lernaea are prevalent throughout the country and further surveys in the rivers of India might lead to the discovery of L. cyprinacea in those areas.

\section{References}

Avenant-Oldewage A. 2012. Lernaea cyprinacea and related species. In: Woo PTK, Buchman K (Eds), Fish parasites, parasitology and protection. CAB International, London, p. 337-349

Blaxter, M. and Floyd, R. 2003. Molecular taxonomics for biodiversity surveys: already a reality. Trends Ecol. Evol., 18: 268-269.

Blaxter, M. 2004. The promise of DNA taxonomy. Philosophical Transactions of the Royal Society of London Series Biol. Sci., 359: 669-679.

Felsenstein, J. 1985. Confidence limits on phylogenies: An approach using the bootstrap. Evolution, 39: 783.

Fratello, B. and Sabatini M. A. 1972. Cariologia e Sistematica di Lernaea cyprinacea L. (Crustacea, Copepoda) Atti Academia Nazionale dei Lincei. Memorie Classe di Scienze Fisische, Matematiche e Naturali, 52(8): 209-213.

Fryer, G. 1961. Variation and systematic problems in a group of lernaeid copepods. Crustaceana, 2: 275-285.

Fryer, G. 1968. The parasitic crustacean of African freshwater fishes, their biology and distribution. J. Zool., London, 156: 45-95.

Gnanamuthu, C. P. 1956. Lernaea bengalensis sp. nov. a copepod parasitic on Channa punctatus. Records of the Indian Museum, 54: 5-8.

Gnanamuthu, C. P. 1951. Lernaea chackoensis n. sp., a copepod parasitic on two Madras fishes. Parasitology, 41: 143-147.

Harding, J. P. 1950. On some species of Lernaea (Crustacea, Copepoda: parasites of freshwater fish). Bull. Br. Mus. Nat. Hist. Zool., 1: 1-27.

Ho, J. S. 1998. Cladistics of Lernaeidae (Cyclopoida), a major family of freshwater fish parasites. J. Mar. Syst., 15: $177-183$.
Hu, Y. T. 1948. Studies on the parasitic copepods of China, Part 3. The far-eastern allies of Lernaea cyprinacea L. with a description of two new subspecies and Lernaea rhodei sp. nov. Sinesia, 19: 86-98.

Kabata, Z. 1979. Parasitic copepoda of the British fishes, The Ray Society, London, 468 pp.

Kaewmongkol, S., Kaewmongkol, G., Inthong, N., Lakkitjaroen, N., Sirinarumitr, T., Berry C. M., Jonsson, N. N., Stich, R. W. and Jittapalapong, S. 2015. Variation among Bm86 sequences in Rhipicephalus (Boophilus) microplus ticks collected from cattle across Thailand. Exp. Applied Acarol., 66(2): 247-56.

Kimura, M. 1980. A simple method for estimating evolutionary rate of base substitutions through comparative studies of nucleotide sequences. J. Mol. Evol., 16: 111.

Larkin, M. A., Blackshields, G., Brown, N. P., Chenna, R., McGettigan, P. A., McWilliam, H., Valentin, F., Wallace, I. M., Wilm, A., Lopez, R., Thompson, J. D., Gibson, T. J. and Higgins, D. G. 2007. Clustal W and Clustal X version 2.0. Bioinformatics, 23: 2947-2948.

Lester, R. G. and Hayward, C. J. 2006. Phylum Arthropoda. In: Woo, P. T. K. and Leatherland, J. F. (Eds.), Fish diseases and disorders, vol. 1: Protozoan and metazoan infections, $2^{\text {nd }}$ edn., CAB International, p. 466-565.

Linnaeus, C. 1758. Systema Naturae. Editio decimal.1. Regnum Animale, Holmiae, Laurentii Salvii, 824 pp.

Monod, T. 1932. Contribution a letude de quelques copepodes parasites de poisons, Annales De Parasitologie Humaine Et Comparee, Paris, 10: 345.

Poddubnaya, A. V. 1973. Variability and specificity of Lernaea parasitic on pond fishes. Trudy Vsesoyouznogo nauchnoissledovalskogo Instituta prudovgo Rybnogo Khozyaystva (in Russian), 22: 159-173.

Poddubnaya, A. V. 1978. Contribution to the knowledge of zoogeography of the crustacean genus Lernaea Linni,1746. Trudy Vsesoyouznogo nauchno-issledovalskogo Instituta prudovgo Rybnogo Khozyaystva (in Russian). 27: 111-124.

Raue, H. A. W., Musters, C. A., Rutgers, J., Van't, R. and Planta, R. J. 1990. rRNA: from structure to function. In: Hill W. E. A., Dahlberg, R. A., Garrett, P. B., Moore, D. Schlessinger and Warner, J. R. (Eds.), The ribosome, structure, function and evolution, American Society of Microbiology, Washington, p. 217-235.

Saitou, N. and Nei, M. 1987. The neighbor-joining method: A new method for reconstructing phylogenetic trees. Mol. Biol. Evol., 4: 406.

Schlotterer, C. 1998. Ribosomal DNA probes and primers. In: Karp, A., Isaac, P. G., Ingram, D. S. (Eds.), Molecular tools for screening biodiversity. Chapman and Hall, London, p. 267-276.

Seenappa, D., Manohar, L. and Shetty, H. P. C. 1980. A new species of Lernaea parasitic on Catla catla (Ham.) and Labeo rohita (Ham.). Mysore J. Agr. Sci., 14: 574-577. 
Thomas, S.1989. Studies on the freshwater copepod fish parasites of Kerala. Ph. D. thesis, Cochin University of Science and Technology, Cochin, India.

Soejanto, S. R. 1965. On the occurrence of a new species of parasitic copepod of the genus Lernaea on a freshwater fish in Indonesia. Treubia Bogor, 26(4): 207-209.

Song, Y., Wang, G. T., Yao, W. J., Gao, Q. and Nie, P. 2008. Phylogeny of freshwater parasitic copepods in the Ergasilidae (Copepoda: Poecilostomatoida) based on $18 \mathrm{~S}$ and 28S rDNA sequences. Parasitol. Res., 102: 299-306.

Srinivasachar, H. R. and Sundarabai, A. 1974. Studies on crustacean parasites of freshwater fishes of Mysore, Part I. Morphology of a new copepod parasite Lernaea hesarghattensis sp. nov. on Lebistes reticulatus (Peters). Proc. Indian Acad. Sci., 80(B): 139.
Tamura, K., Peterson, D., Peterson, N., Stecher, G., Nei, M. and Kumar, S. 2011. MEGA5: Molecular evolutionary genetics analysis using maximum likelihood, evolutionary distance and maximum parsimony methods. Molecular Biol. Evol., 28: 2731-2739.

Tautz, D., Arctander, P., Minelli, A., Thomas, R. H. and Vogler, A. P. 2002. DNA points the way ahead in taxonomy. Nature, 418: 479.

Tautz, D., Arctander, P., Minelli, A., Thomas, R. H. and Vogler, A. P. 2003. A plea for DNA taxonomy. Trends in Ecol. Evol., 18: 70-74.

Yu, S. C. 1938. Some parasitic copepods from the freshwater fishes of China. Bull. Fan Mem. Inst. Biol., Zool. Ser. 8(2): 105. 